B.

Eberhard Geisler

(Mainz)

\title{
MICHAUX REIST NACH ECUADOR, ODER EINE ANDERE EXOTIK
}

Zwei seiner Reisen hat Michaux in Büchern festgehalten: 1927 reist er nach Ecuador, um schließlich über den Amazonas und die brasilianische Atlantikküste nach Europa zurückzukehren, eine Reise, von der er 1928 Notizen unter dem Titel Ecuador veröffentlicht; 1930/31 fährt er nach Indien, China, Japan und Malaysia, um 1933 den Band Un barbare en Asie vorzulegen. Beide Werke stehen diametral zueinander: Wird Ecuador vom Autor großenteils mit Skepsis, Gleichgültigkeit und Ablehnung bedacht, so bezeichnet er die Reise in den Fernen Osten als seine erste wirkliche Reise »enfin son voyage ${ }^{1}$ - und die Völker des Ostens als die ersten, die er ernstnehmen kann ${ }^{2}$. Wenn ich mich im Folgenden ausschließlich dem Band Ecuador zuwende, dann nicht allein des lateinamerikanischen Schwerpunkts dieses Symposions wegen, sondern vor allem auch weil sich an diesem Buch beobachten lässt, wie das, was Michaux' Werk ausmachen sollte, sich nicht zuletzt in der kritischen Auseinandersetzung mit der traditionellen Reiseliteratur entwickelt hat. »Der kürzeste Weg zu sich selbst führt um den Globus«, schreibt Hermann von Keyserling ${ }^{3}$, und es ist der Text, der anlässlich von Michaux' Lateinamerika-Reise entsteht, in dem sich wie in einem Vorgriff die wichtigsten Aspekte seines Gesamtwerks gespiegelt finden.

Der Autor lässt keinen Zweifel daran, dass er Lesererwartungen im Sinn des Exotismus des 19. Jahrhunderts nicht mehr zu erfüllen bereit ist. Ähnlich wie Victor Segalen, André Gide und Michel Leiris, die in ihren

Henri Michaux $\left({ }^{2}\right.$ 1983: 14)

Henri Michaux (1982: 13).

Zitiert bzw. rückübersetzt nach Jean-Marc Moura (1998: 329). 
ethnographischen Texten und Reisebeschreibungen die naive Verklärung der Ferne durch den Hinweis auf die Wirklichkeit des Kolonialismus zu entzaubern versuchten, bemüht sich auch Michaux um eine größere Nähe zur Realität. Er bedient sich dabei zweier Argumente. Das erste ist die Theorie des fremden Alltags. Die Welt teilt sich nicht mehr in den ennui der Heimat einerseits und das Klischee von der farbigen Tropenwelt andererseits auf, sondern bietet unterschiedliche, in ihrer Eintönigkeit und Banalität wohl aber verwandte Zonen. Auf den Reisenden wartet überall ein fremder und stets ähnlich beschwerlicher Alltag. Am Amazonas machen verschiedene unangenehme Lebewesen diesen Alltag aus: eine Art Blutegel, der sich an die Beine heftet, Mücken, die sich in den Augenwimpern niederlassen, ein wollfadendünner Fisch, der in die Körperöffnungen eindringt und beim Wiederaustritt Blutungen hervorruft, schließlich kleine, jedoch Menschenfleisch fressende Fische. Auch die stundenlange Fahrt in der Piroge, einem schmalen, mit einem Blätterdach versehenen Einbaum, der keine Bewegung des Reisenden und nur den starren Blick nach vorne gestattet, ist eine einzige Tortur und hat mit der Vorstellung von Freiheit, die der Europäer mit diesem Gefährt verbinden mag, wenig zu tun. »[V]ous réclamez du tigre, du puma, mais on ne vous donne que du quotidien. $\ll^{4}$ Das zweite Argument besteht im Hinweis auf die mittlerweile hundertprozentige verkehrstechnische und touristische Erschlossenheit der Welt, auf der es nichts mehr zu entdecken gibt. Ähnlich wie Paul Morand drei Jahre zuvor in Rien que la terre stellt Michaux fest:

Non, je l'ai déjà dit ailleurs. Cette terre est rincée de son exotisme. Si dans cent ans, nous n'avons pas obtenu d'être en relation avec une autre planète (mais nous y arriverons) l'humanité est perdue. (Ou alors l'intérieur de la terre?) Il n'y a plus moyen de vivre, nous éclatons, nous faisons la guerre, nous faisons tout mal, nous n'en pouvons plus de rester sur cette écorce. Nous souffrons mortellement; de la dimension, de l'avenir de la dimension dont nous sommes privés, maintenant que nous avons fait à satiété le tour de la terre. (EJV, 35$)^{5}$

Wir werden sehen, welche alternativen Formen und Dimensionen des Exotischen Michaux in diesem Buch entwickelt.

4 Henri Michaux (1984: 160); Verweise auf diese Ausgabe erscheinen im Text unter Angabe der Sigle EJV (Ecuador. Journal de voyage) und der entsprechenden Seiten.

5 Paul Morand beurteilt die Situation nach dem obsolet gewordenen Exotismus ähnlich dramatisch: »Il restera d'entrer à la Trappe, - cette légion étrangère de Dieu, - et de chercher désormais en hauteur un infini que l'étendue ne peut plus nous donner, ou d'aller conquérir d'autres planètes«, in: Paul Morand (1926: 12). 
Jahre später - 1938 - wird Michaux einer Sammlung von Prosa und Lyrik den Titel Lointain intérieur geben. Damit ist sein Projekt einer eigenen neuen Exotik treffend bezeichnet. Was er der traditionellen Exotik zunächst entgegensetzt, ist eine radikale Subjektivität. Diese erweist sich vor allem in einem gesteigerten Vermögen zu Negativität. »Aucune contrée ne me plaît: voilà le voyageur que je suis« (EJV, 41). Um diese Subjektivität als Zentrum seiner Welt bzw. Reflexionen zu verdeutlichen, ist er auch bereit, sich auf provokante Weise vom romantischen Bild des Indios bzw. vom Bild des Guten Wilden zu distanzieren. Er notiert in sein Tagebuch, dass er den Brief eines Bekannten erhalten hat, der in einem Berliner Museum gerade in Wachs gegossene Indios gesehen hat und nun begeistert schreibt, diese steckten voller Poesie, und Michaux werde sie bei seiner Abreise aus Ecuador bestimmt vermissen. Michaux bemerkt dazu:

«Indien», «Indien», vous voulez me stupéfier avec ça. Un indien, un homme quoi! Un homme comme tous les autres, prudent sans départs, qui n'arrive à rien, qui ne cherche pas, l'homme "comme ça».[...] Une fois pour toutes: les hommes qui n'aident pas à mon perfectionnement: zéro. (EJV, 98)

Gewiss lässt er den Indios auch Gerechtigkeit widerfahren, indem er einen anderen Bekannten zitiert, der darauf hinweist, dass nur diejenigen Indios ihr Lachen bewahrt haben, welche die Kolonialisation nicht an ihrem eigenen Leib erfahren haben, oder auch indem er einzelne Beschreibungen der indianischen Welt abgibt, aber es gibt hier kein ethnographisches Pathos, das im Vordergrund stünde oder durchgängig wäre. Anders ist der Fall bekanntlich bei Michel Leiris, der in seinen Expeditionsbericht L'Afrique fantôme von 1934 zwar auch Subjektivität einfließen, das ethnographische Interesse aber - trotz aller Zweifel, die er an seiner ethnologischen Perspektive hegt - im Vordergrund lässt.

Der Bedeutung der Subjektivität entsprechen längere, großenteils in lyrischer Form abgefasste autobiographische Passagen. Auch mit dieser Tendenz bildet Ecuador das Gesamtwerk in nuce ab. Wenn Helmut Heißenbüttel von Michaux als einem »Literaten der Selbstentblößung « sprach $^{6}$, dann wird in dieser Formulierung noch der Schock spürbar, der den Leser ereilen mag, der diesen Autor zum ersten Mal liest und neben dessen Beschreibungen objektiver Realität subtile Texte psychischer wie physiologischer

6 Helmut Heißenbüttel (1966). 
Innenschau findet. Der Autor leidet an Herzinsuffizienz, und die dünne Luft der Anden und die sonstigen Strapazen machen ihm besonders zu schaffen. Er habe ein Hühnerskelett, schreibt er (EJV, 46), das für die Erschöpfungen und Fieberanfälle der Reise nicht gemacht sei; diese wird, wie man sieht, zu einem gigantischen Selbstversuch. Darüber hinaus lastet auf ihm das elterliche Verdikt des Versagers (EJV, 66). In seiner Kindheit befand man, dass er für ein Studium nicht tauglich sei (EJV, 81). Tatsächlich brach er sein Medizinstudium ab, um schon vor der Reise nach Ecuador als Matrose über den Atlantik zu schippern. In Ecuador überlegt er, ob er seinen Eltern nicht einen Brief schreiben solle, in dem er sich als großer Abenteurer darstellt und mit seinen Erlebnissen prahlt - »J'habite une cabane de bambous, soutenue par des troncs de palmiers. Un tigre a mangé un mulet ici la nuit dernière, etc." (EJV, 66) -, aber er entscheidet sich dann doch, den Brief ungeschrieben zu lassen, für den Fall, dass sich doch noch etwas ereignen sollte, was die Skepsis der Eltern ihrem Sprössling gegenüber bestätigen würde. Besonders bemerkenswert für die Selbstanalyse des Autors ist das in diesem Band enthaltene Gedicht »Je suis né troué«. Der Autor nimmt ein Loch in seiner Brust wahr, das sich immer wieder mit der Empfindung großer Leere, aber auch von Mangel, Hass und Ohnmacht verbindet. Für diese seelische Verfasstheit gibt es keinerlei Abhilfe, er muss immer wieder zu diesem Loch zurückkehren, und auch die Erotik, wie er ausdrücklich vermerkt, kann diesen Mangel nicht füllen. Für das fehlende Fundament seines Ich hat er eine anschauliche Metapher gefunden: »Je me suis bâti sur une colonne absente« (EJV, 95). Auch wenn hier eine konkrete Individualität zum Ausdruck kommt, so bildet diese jedoch die Ausgangsposition für Auslotungen menschlicher Befindlichkeiten allgemein und lässt Texte entstehen, die in Parallele zu modernen Theorien des Subjekts zu sehen sind, welche im Subjekt weniger eine autonome Instanz als vielmehr eine immer schon in Welt eingebundene Konstruktion erkennen. Das Ich steht hier vor der paradoxen Aufgabe, sich in der Abgründigkeit der Existenz einen Halt suchen zu müssen.

Die Suche nach einer anderen Exotik führt in dem hier vorgestellten Buch auch zu einer eigenen Form von Wahrnehmung und Beschreibung äußerer Welt. Der zentralen Rolle der Subjektivität steht damit auch eine Bemühung um Darstellung von Alterität gegenüber. Der Autor unterwandert mit seiner Kritik des Exotismus zwar das Terrain der Philanthropie, besorgt dabei aber nicht das Geschäft des Eurozentrismus. Er befindet sich 
in einem indianischem Land, aber er bemüht das Auge des Schwarzen, um eine alternative, nicht-europäische Form der Apperzeption zu verdeutlichen:

Le nègre a dans sa tête une étrange expression. Comme les orangs-outangs. Et les orangs ont des yeux très humains. Le nègre: une eau dans la figure, c'est son œil.

Les blancs paraissent avoir dans les yeux un noyau plus au moins grand suivant les individus. Ce noyau jamais ne se dissout en regard. Il est la marque du secret, du phénomène cérébral, de la réflexion insoluble en physionomie. (EJV, 26)

Der Unterschied zwischen dem Blick der Weißen und dem der Schwarzen wird hier durch den Gegensatz von Starrheit und Beweglichkeit markiert. Der Blick des Europäers ist vom Geheimnis seiner nicht nach außen tretenden Reflexion bestimmt, im Auge des Schwarzen scheint sich dagegen ein Verhältnis von Subjekt und Objekt auszudrücken, das noch nicht von Fixierung bestimmt ist bzw. sich noch im Fluss befindet. Wenn der Blick etwas ist, was sich gleichsam an die Dinge verschenkt, dann muss das abendländische Subjekt hier konsequenterweise als blicklos erscheinen. Der Schwarze umgekehrt scheint zu blicken, weil sein Blick noch präobjektiv ist.

Eine mögliche literarische Realisierung dieser Wahrnehmung von Welt dürfte darin bestehen, den »mimétisme des choses«, von dem Michaux spricht, sichtbar zu machen. Unter diesem Begriff ist eine anthropomorphe Sicht der Wirklichkeit zu verstehen, bzw. ein in die sogenannten unbelebten Dinge projiziertes Vermögen, sich zu verwandeln und als Wesen zu erscheinen. Der Autor merkt an, dass er diese Sicht der Dinge nach langer Zeit für sich wiederentdeckt hat:

Le mimétisme m'a longtemps paru un de ces attrape-savants comme il en existe tant. Souvent, je sentis par ailleurs, et aujourd'hui ma virginité de vue, d'observation refaite pour ainsi dire, je constate une fois de plus, le mimétisme des choses, des objets, dits inanimés. (EJV, 27)

Auch hier geht es im Gefolge phänomenologischer Vorstellungen nicht um Objektivität an sich, sondern um die Darstellung der Resonanz von Welt im Subjekt. Nicht von ungefähr erinnert dieser »mimétisme« an eine kindliche Sicht der Dinge; in seinem späten Prosastück Les commencements. Essais d'enfants, dessins d'enfants von 1983 zeigt Michaux auf - wie vor ihm üb- 
rigens auch schon Merleau-Ponty ${ }^{7}$-, dass gerade auch die Kinderzeichnungen keine maßstabgetreue und vollständige Wiedergabe der Gegenstände suchen, sondern den Gestus der ersten Annäherung an diese. Wenn Michaux trotz seiner Negativität in einer Vielzahl von Skizzen einen lebendigen Eindruck seiner Reise, von Menschen und Landschaften Lateinamerikas vermittelt, dann verdankt er dies weitgehend dem Verfahren des »mimétisme«. Im Folgenden möchte ich drei Beispiele geben:

1. Eine Darstellung dreckiger und altersschwacher Schiffe wird mit folgenden Worten gegeben:

La plupart des navires, si sales, infects, et rouillés qu'on se demande comment la mer arrive à trouver la quille et que c'est un navire, et pas un détritus ou un effet de la lumière, et au bout de combien de temps elle y arrive, après quels tâtonnements, quelles réflexions, quels commentaires. (EJV, 28)

2. Der ecuadorianische Urwald wird wie folgt präsentiert:

Arbres des tropiques, à l'air un peu naïf, un peu bête, à grandes feuilles, mes arbres! La forêt tropicale est immense et mouvementée, très humaine, haute, tragique, pleine de retours vers la terre. Les parasites veulent bien s'élever. Ils choisissent un arbre, mais après avoir pris quelque hauteur, les voici tous qui bêlent et reserpentent vers la terre. (EJV, 60)

3. Der Norden Ecuadors lässt sich verlauten:

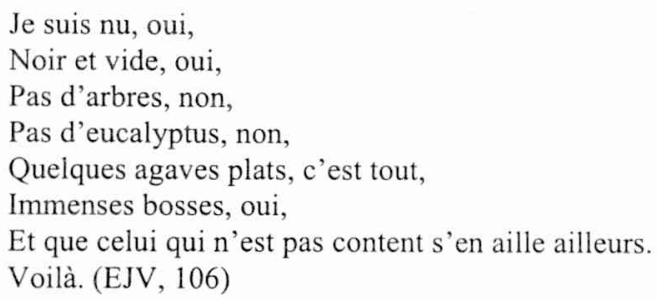

Vor allem letztes Zitat kann deutlich machen, dass Michaux bei seinem Versuch, einen präobjektiven Zugang zur Welt zu finden, immer wieder auch den eigenen literarischen Diskurs von dieser Präobjektivität durchdringen und bestimmen lässt. Ähnliches gilt für den in seinem Werk vielfach entfalteten Motivkreis der Kindheit. ${ }^{8}$ Diese ist die Phase eines - sich

7 Maurice Merleau-Ponty (1969). - Zur Nähe zwischen Michaux und Merleau-Ponty siehe auch Raymond Bellour (1986: 222-223).

8 Étienne Rabaté (1987). 
gerade in den Kinderzeichnungen erweisenden - uranfänglichen Verhältnisses des Subjekts zur Welt und zugleich der Raum eines uranfänglichen Sprechens. Hierhin gehört sowohl die in Michaux' Texten immer wieder beschworene Magie, die ein Weltverhältnis vor der Trennung zwischen Subjekt und Welt zum Ausdruck bringt: »J'étais autrefois bien nerveux. Me voici sur une nouvelle voie: Je mets une pomme sur ma table. Puis je me mets dans cette pomme. Quelle tranquillité! «, sowie auch eine Anzahl von Gedichten, die den kindlichen Erwerb der Sprache stammelnd und spielerisch in Szene setzen:

Certains textes $[\ldots]$ sont de véritables archéologies du langage; ils reconstituent l'apprentissage qu'en fait l'enfant, le montrent en formation, des borborygmes aux premiers substantifs maladroits, sur lesquels se greffent des adjectifs, puis des prépositions, jusqu'au développement sophistiqué des phrases complexes. ${ }^{10}$

Der Lust am kindlichen Spracherwerb steht aber, wie wir noch sehen werden, eine tiefe Skepsis der entfalteten Sprache gegenüber.

Victor Segalen hat in seinem Roman Les Immémoriaux (1907) bekanntlich die traditionelle Sichtweise der exotischen Literatur aufgehoben, indem er sie umgekehrt hat: Er schildert dort am Beispiel der europäischen Eroberung und Missionierung Tahitis nicht mehr die Reaktion des Reisenden auf die Fremde, sondern die Reaktion des fremden Milieus auf den Reisenden. Michaux verfährt in einer Passage ähnlich, wenn er auf die Millionen von Fischen aufmerksam macht, die den Kiel des Schiffes gesehen haben, auf dem der Autor den Atlantik überquert. Man weiß nicht, was die Fische bei diesem Anblick gedacht haben mögen. Michaux' Europa-Kritik ist hierbei nicht ethnographisch bzw. kolonialkritisch motiviert, sondern richtet sich allgemeiner gegen die Folgen einer unbefragten Naturbeherrschung. Ingenieure und Geschäftsleute, die Vorhut Europas, sind im Verfolg ihrer Zwecke nur daran interessiert, das Meer rasch zu überqueren. Ihr Prinzip lautet (und so macht Michaux es ihnen zum Vorwurf): »Toujours se mettre audessus de la nature jamais dedans « (EJV, 18). Deshalb ist das Schiff blind und blöde, und man könnte die Reise, heißt es, genauso gut in einem Sack vornehmen. An diese Feststellung knüpft sich die Prophezeiung einer neuen Exotik: In weniger als fünfzig Jahren werde man alle Schiffe mit Gerä-

9 Henri Michaux (1983: 9).

10 Étienne Rabaté (1987: 122). 
ten ausrüsten, die den Reisenden zur unterseeischen Welt in Beziehung treten ließen. Das Gesichtsfeld würde sich damit über den engen Rahmen der Zweckrationalität hinaus öffnen. An dieser Stelle sei kurz noch auf das Reisetagebuch eines anderen Schriftstellers verwiesen. Ein Jahr vor Ecuador legte André Gide seinen Band Voyage au Congo vor. An einer Stelle spricht Gide - der mit seiner Rede Découvrons Henri Michaux von 1941 übrigens zu den Entdeckern unseres Autors zählt - von der Angst, die ihn bei der Befahrung des Kongoflusses befällt. »Chaque occasion de descendre à terre nous trouve prêts « ${ }^{11}$, und weiter:

Lente remontée du fleuve dans la nuit. Sur la rive gauche, au loin, quelques lumières; un feu de brousse, à l'horizon; à nos pieds l'effrayante épaisseur des eaux. $^{12}$

Gides Notiz hält den Schrecken über die Abgründigkeit fest, die normalerweise hinter dem Eindruck der Sicherheit von Naturbeherrschung verborgen bleibt. Die undurchdringlichen Gewässer gemahnen an eine Natur, die sich nicht immer domestizieren lässt. Michaux erkennt in der Abgründigkeit dagegen den Normalfall und richtet sich gleichsam in ihr ein. Ich verweise hier nur auf die beiden Prosastücke »Conseil au sujet de la mer « aus La Nuit remue (1935) sowie »Ma vie s'arrêta" aus Entre centre et absence (1936), die beide als Kontrast zu Edgar Allan Poes Erzählung vom Malstrom gelesen werden können, insofern der Amerikaner noch die Möglichkeit einer listigen Überwindung der Natur gegeben sieht, Michaux aber die Abgründigkeit als unaufhebbar und nahezu als seelischen Alltag begreift. ${ }^{13}$

An die Stelle der traditionellen Exotik tritt bei diesem Autor die Wendung auf das Bewusstseinsleben. In diesem Sinn kann er in sein Tagebuch notieren: "Je ne suis plus à Quito, je suis dans la lecture« (EJV, 41). Zu dieser radikalen Wendung zum Bewusstseinsleben hin gehört auch das Lob der Tapete bzw. der Wand. Kurz nach seiner Ankunft in Ecuador beobachtet sich der Autor, wie er sich in Risse, Flecken und Linien einer Wand versenkt und dabei unerwartete Entdeckungen macht. Er sieht ein mit Gesang begabtes Möbelstück, die Figur eines Narren sowie eine Frauengestalt (EJV, 36-37). Diese Szenerie ist kaum zu interpretieren (für sein Werk gilt schließlich insgesamt, dass die hermeneutische Funktion immer wieder in

11 André Gide $\left({ }^{60} 1947: 18\right)$.

12 Ibid.

13 Siehe hierzu Eberhard Geisler (1992: 182-184). 
den Hintergrund rückt und subjektiven, großenteils exorzistischen Funktionen Platz macht); es kommt hier hauptsächlich wohl darauf an, überhaupt auf das Vermögen der Imagination aufmerksam zu machen. An anderer Stelle in diesem Buch bezeichnet Michaux den Blick auf die Wand als besonders effizienten Weg zur Selbsterkenntnis; seine Reise, bekennt er, war insofern ein Fehler: »On trouve aussi bien sa vérité en regardant quarantehuit heures une quelconque tapisserie de mur« (EJV, 120). Interessanterweise äußert sich auch André Gide in dem bereits zitierten Tagebuch seiner Kongoreise zu dem - übrigens auf Leonardo da Vinci zurückgehenden ${ }^{14}$ Vorschlag, statt der gewohnten farbigen äußeren Wirklichkeit einmal nur eine Wand zu betrachten. Diese Äußerung findet sich anlässlich seiner unterwegs vorgenommenen Lektüre der Grabrede Bossuets auf Henriette d'Angleterre. Obwohl Gide dieses Stück Literatur schätzt, wendet er sich offenkundig gegen Bossuets Betonung der Unabänderlichkeit der Endlichkeit menschlicher Existenz einerseits, ihrer Unsterblichkeit andererseits. Anders als bei Michaux, bei dem der Blick zur Wand eine interessante Alternative zur >normalen< Wirklichkeitsschau darstellt, wertet Gide ihn darum ab als Blick auf das Unabänderliche und plädiert für die Anschauung der abwechslungsreichen äußeren Wirklichkeit als ein dem lebendigen Subjekt einzig Gemäßes. Gide antwortet entrüstet auf den Vorschlag einer solchen Wand-Vision:

Imagine-t-on quelqu'un qui dirait à un voyageur: «Ne regardez donc pas le fuyant paysage, contemplez plutôt la paroi du wagon, qui elle, du moins, ne change pas.» Eh parbleu! lui répondrais-je, j'aurais tout le temps de contempler l'immuable, puisque vous m'affirmez que mon âme est immortelle; permettezmoi d'aimer bien vite ce qui disparaîtra dans un instant. ${ }^{15}$

Bei Gide steht die Wand für Objektivität, und zwar für eine unerwünschte; bei Michaux ist sie willkommener Projektionsschirm seines Innenlebens. In die Nähe dieses Phänomens gehört auch der Tagtraum. Der Autor berichtet

14 Unter dem Titel »Modo d'aumentare e destare lo 'ngegnio a varie invenzioni« notiert Leonardo in seinen Schriften über die Malerei: „Non resterò però di mettere infra questi precetti una nova invenzione di speculazione, la quale, benchè paia piccola e quasi degna di riso, non di meno è di grande utilità a destare lo 'ngegnio a varie invenzioni: e questo è, se tu riguarderai in alcuni muri imbrattati di varie macchie o pietre di vari misti, se avrai a invencionare qualche sito, potrai li vedere similitudine di diversi paesi, ornati di montagne, fiumi, sassi, alberi, pianure, grandi valli e colli in diversi modi; ancora vi potrai vedere diverse battaglie e atti pronti di figure, strane arie di volti e abiti e infinite cose, le quali tu potrai ridurre integra e bona forma«, Leonardo da Vinci (1928: 132).

André Gide $\left({ }^{60} 1947: 30-31\right)$. 
von seiner Praxis, vor dem Schlafengehen sich in angenehme und vorteilhafte Situationen hineinzudenken, um Schlaf bzw. eine Bastion gegen die Aggressivität der anderen zu finden (EJV, 47). In seinem Buch Façons d'endormi, façons d'éveillé von 1969 ist er nochmals auf das Phänomen des Tagtraums zu sprechen gekommen, um ihn auch dort als Weg zur individuellen Freiheit zu apostrophieren.

Ecuador ist, wie eingangs angedeutet, eine Art Musterbuch, in dem sich die wesentlichen Aspekte des Gesamtwerks bereits vertreten finden. So erwähnt Michaux auch seine Malerei, für die er später ebenso bekannt wie für seine Dichtung werden sollte, und zwar hinsichtlich der menschlichen Phantasmen, Monster und Seelenporträts, die er seit etwa 1925 malt und auch in Ecuador dem Gedanken an die Darstellung äußerer Wirklichkeit entgegensetzt, und so erwähnt er hier auch Experimente mit Drogen, die er später in mehreren Büchern und lyrischen Texten genauer erkunden sollte.

Ein weiterer Aspekt ist das Lob der Eintönigkeit. Einer der hier gesammelten Texte setzt bei dem Versuch an, die Kordilleren zu beschreiben. Der große Gebirgszug stellt sich in seiner Nacktheit und mangelnden Vegetation als nichts anderes denn als aufgehäufte Erde dar, als unablässige Wiederholung von Erde. Dem Autor wird diese Beobachtung zum Anlass, über Wiederholung und Eintönigkeit nachzudenken, die ihm entgegen landläufiger Auffassung als ebenso wertvoll wie die Vielfalt erscheinen. Die Kordilleren sind - ähnlich wie das Meer oder ein Wald - Beispiel für eine Größe, die nur schwer optisch zu erfassen und sprachlich auszudrücken ist. Die Philosophen, die zuhause blieben und hinter der Vielfalt des Seienden immer wieder nur das Sein erkennten, seien der Größe näher als die Reisenden, die sich unterwegs immer wieder nur innerhalb der Mannigfaltigkeit bewegten.

Il est superflu de constater combien les voyageurs, quand ils écrivent, sont dépourvus de grandeur (causes et effets sont mêlés ici) et combien celle-ci est courante chez les philosophes, qui connaissent si peu la terre. On en trouve parmi eux qui, tellement pris de cette passion de la répétition, ont fini par ne plus voir que l'être en chaque être et y arrivent de bonne foi. Sa femme, un chien, un hibou, un saule: être, être, être. Il voit leur différence, mais l'être répété l'enivre par-dessus toute différence. (EJV, 184-185)

Michaux selbst hat in seinem lyrischen Werk der Monotonie einen großen Raum zugebilligt. In den litaneihaften Wiederholungen vieler seiner Gedichte wird die bedrohliche Umwelt gleichsam zersungen, um jenseits der 
Mannigfaltigkeit dem lyrischen Ich ein Aufgehen in einer mystischen Einheit zu ermöglichen. In diesem Sinn urteilt auch Henri Meschonnic:

La référence au sacré, et à la magie, est explicite. C'est parce que la visée de Michaux déborde la sécularisation esthétique, ou esthétisante, d'autres préceptes de répétition, comme celui de Gertrude Stein. C'est une visée éthique. Proche du mythe, en tant que récit de vérité. ${ }^{16}$

Eine Passage aus Ecuador zeigt dabei die Genese der Repetitivität als letztem Rekurs eines Ich, das seine Schwäche und Not nicht anders als am Rande einer entfalteten Diskursivität zum Ausdruck bringen kann:

Prêtez-moi de la grandeur,

Prêtez-moi de la grandeur,

Prêtez-moi de la lenteur,

Prêtez-moi de la lenteur,

Prêtez-moi tout,

Et prêtez-vous à moi,

Et prêtez encore,

Et tout de même ça ne suffira pas. (EJV, 148)

Die Wiederholung zerreibt die Sprache, weil innerhalb der Sprachkette und selbst wenn dort >alles` genannt würde - nichts erscheinen kann, was den bodenlosen Mangel des Ich aufzufüllen vermöchte. Ähnlich formuliert der Autor anlässlich eines Experiments, das er in späteren Jahren mit einem schlichten, mit Eisenlamellen bestückten afrikanischen Musikinstrument (Sanzas) durchgeführt hat. Er entlockt diesem Instrument einen monotonen, kläglichen Laut:

Cependant je faisais à l'instrument répéter et répéter le signal de désolation, ce son dévastateur pour moi sauveur, l'expression têtue du "sans espoir», je l'écoutais avec avidité. Pas de discours. Pas d'enchaînement. Seulement dénégation sur dénégation. Un unique son rébarbatif. Il suffisait. ${ }^{17}$

Genügt dem Ich innerhalb der diskursiven Kette nichts, so kann es Genüge am Rand der Sprache (hier: der musikalischen Sprache) finden (»Il suffisait«). Diese Monotonie ist das Medium einer tiefen Berauschung. In Ecuador notiert der Autor:

16 Henri Meschonnic (1987: 197).

17 Henri Michaux (1985: 28-29). 
Ma chambre donne sur un volcan.

La fenêtre de ma chambre donne sur un volcan.

Enfin un volcan.

Je suis à deux pas d'un volcan.

Il y avait dans notre propriété un volcan.

Volcan, volcan, volcan.

C'est ma musique pour ce soir.

[...] Peu de phrases. Le gong fidèle d'un mot. (EJV, 46-47)

Hier ist die Sprache als System von Differenzen weitgehend stillgelegt, und die eintönige Lautfolge kann als eigentliche Musik aufgefasst werden, mit deren Hilfe er das Reich der inneren Exotik zu betreten und sein Zentrum am Rand (der Sprache wie von Intersubjektivität überhaupt) zu finden vermag. Die Eintönigkeit konfiguriert dabei das Paradox, dass die Absage an Sprache noch einmal sprachlich mitgeteilt wird.

Die Einheit steht auch im Vordergrund des lyrischen Spätwerks, das zu einem gewissen Teil auf Erfahrungen mit Drogen beruht. Auch wenn der Autor dort - ähnlich wie auch in seiner Tuschmalpraxis, deren »[G]estes du dépassement / du dépassement / surtout du dépassement $«{ }^{18}$ er hymnisch feiern wird - etwas wie reine Heterogenität zu erfahren beansprucht, rangiert die Einheit - wie man vor allem hinsichtlich bestimmter später Texte kritisch einwenden kann - faktisch trotzdem vor der Mannigfaltigkeit. Eine ähnliche Aporie findet sich bekanntlich in der zeitgenössischen Philosophie - bei Bergson, der in der »durée« bzw. im »élan vital« ebenso Einheit und Mannigfaltigkeit ineins zu denken beanspruchte, der Einheit aber letztlich den Vorrang einräumen musste. ${ }^{19}$

Die Wendung gegen die Sprache, die in der repetitiven Litanei impliziert ist, wird in unserem Text auch noch in anderer Hinsicht deutlich. Der Autor bekennt, er sei ein schlechter Leser, alle Texte seien für ihn unverständlich, und aus diesem Grund habe er auch für Texte kein Gedächtnis. Neben dieser Leseschwierigkeit verspüre er aber auch das Bedürfnis, die Texte selbst unlesbar zu machen und zu entstellen. Anlass für diesen Tagebucheintrag ist die Lektüre eines Textes eines gewissen Zeixe Man über ei-

18 Henri Michaux ( ${ }^{2} 1982$ [1951]: [41]).

19 Auch Paul Morand übrigens erweist sich als Zeitgenosse Bergsons und stellt die Erfahrung reiner Heterogenität ins Zentrum seines Strebens: »Je m'aperçois encore ici, au moment de débarquer, que je n'aime pas les voyages, que je n'aime que le mouvement. C'est la seule vérité, la seule beauté. Je n'aurai pas honte de ma vie tant qu'elle sera mobile. Seul point fixe: l'idée de changement«, in: Paul Morand (1926: 31-32). 
nen Maler namens Papazoff. Da sich weder Papazoff noch Zeixe Man ermitteln lassen, liegt die Vermutung nahe, dass Michaux' entstellende >Lektüreく sich hier auf Gestalt und Werk Mallarmés bezieht, der zu Beginn der betreffenden Passage genannt wird. ${ }^{20}$ Michaux ergötzt sich daran, das Material seiner >Lektüre $<$ zu entstellen und zu Nonsens werden zu lassen. Ich zitiere drei Sätze:

Après son mariage, son instinct le fit geindre Mallarmé.

Sa pose et son goût des frictions ne facilitèrent pas son abcès.

Geindre était pour lui un homme qui n'avait pas besoin de «self», un roteur obscur enregistrant les actes de naissance. (EJV, 71-72)

Hier wird die bei Michaux immer wieder zu beobachtende Tendenz deutlich, sich in einen Raum jenseits von Sinnhaftigkeit und Intersubjektivität zu begeben. ${ }^{21}$ Eine ähnliche Entstellung von Sprache hat er übrigens später nochmals in dem literarischen Text »Une vie de chien « aus der Sammlung Mes Propriétés beschrieben, wobei im Unterschied zu der Tagebucheintragung auch hier das Paradox eines Vortrags im Plauderton einerseits und der Aufkündigung der intersubjektiven Funktion der Literatur andererseits hervortritt. ${ }^{22}$ An dieser Stelle sei nochmals an Gide erinnert, der ganz im Gegensatz zu Michaux sein Vergnügen an der metonymischen Wucherung der Sprache findet und seinen Reisebericht mit Fußnoten, Lesefrüchten und Kommentaren versieht. Michaux hat sein literarisches Prinzip an anderer Stelle formuliert: »Attention au bourgeonnement! Écrire plutôt pour courtcircuiter. $\ll{ }^{23}$

In Anbetracht des Michauxschen Versuchs einer Herauswindung aus Traditionen und Zusammenhängen sei abschließend - wie zum Teil bereits angedeutet - darauf hingewiesen, dass der Autor trotz aller Randgänge durchaus an bestimmte literarische bzw. philosophische Projekte von Vorläufern und Zeitgenossen anknüpft. Den antirhetorischen Impuls teilt er mit der gesamten Avantgarde. Breton beispielsweise hatte 1920 in einem dadaistischen Manifest geschrieben, der zeitgenössische Dichter zeichne sich

20 In diesem Sinn siehe auch Jean-Pierre Martin (1991).

21 Jean-Pierre Martin (1991: 405) sieht in diesen Sätzen zwar gewisse rekonstruierbare Sinnelemente (Anspielungen an Mallarmé und dessen Werk) gegeben, unterstreicht vor allem aber auch, dass Michaux' Negativität sich hier gegen die Konvention der Lektüre selbst wendet und damit über Mallarmés Hermetik hinausgeht.

22 Cf. hierzu Eberhard Geisler (1992: 99ss.).

23 Henri Michaux (1980: 44). 
durch seine Attacke auf die Sprache als der schlimmsten aller Konventionen aus. ${ }^{24}$ Negativität als Schreibimpuls lernt Michaux bei Lautréamont kennen. Hinsichtlich seines Projekts einer Überbietung der traditionellen Exotik ist als Vorläufer natürlich Rimbaud zu erwähnen, für den die Aufgabe des Dichters weniger in der Kopie vorgegebener Wirklichkeit als vielmehr im aktiven Überschreiten gewohnter Orientierungen im »dérèglement de tous les sens « liegt. Dichtung soll weniger als Resultat denn vielmehr als permanente, Krankheit, Wahn und Droge als Erkenntnismöglichkeiten nutzende Suche nach einem außerhalb der gesellschaftlich sanktionierten Alltagswirklichkeit vermuteten Wesentlichen begriffen werden. ${ }^{25}$ Gegenüber der von Michaux mit Rimbaud anvisierten anderen Exotik bleiben selbst die ehrwürdigsten Werke der Weltliteratur bloß konventionell:

Des personnes sévères disent hardiment de Platon qu'il est médiocre, et Saint Augustin idem, médiocre, et Shakespeare et Dante et Goethe, et tout qui écrivit. D'autres, par contre, envient les écrivains. Mais ils ont tort et grandement. Ceux qui ont écrit, c'est que ça les contentait.

Pour ceux qui n'écrivent point, c'est qu'ils n'ont pas été touchés suffisamment. Peut-être ils sont nés pour plus grand, pour plus beau; et peut-être qu'ils écriraient seulement, si morts, ou devenus coqs ou lamas ou vautours, ils revenaient ensuite à la vie d'homme, ou après quelque séjour infernal ou planétaire, au retour enfin d'une grande aventure et autrement essentielle que la nôtre. (EJV, 7273)

Gleichzeitig, und damit kommen wir zur letzten Passage, die ich hier zitieren möchte, übt sich Michaux in Ecuador aber auch in der Abbildung der auf Reisen erfahrenen Welt. Wie gesagt, bedient er sich des »mimétisme«, um äußere Wirklichkeit zu beschreiben. Neben dem Faszinosum der Einheit, das wir festgestellt haben, findet sich auch die Bereitschaft, die vielfältigen äußeren Dinge zu betrachten und zu beschreiben, und die sich mit dem zweiten Reisebuch, Un barbare en Asie, das in den Fernen Osten führen wird, noch weiter steigert. ${ }^{26}$ In der hier interessierenden Passage greift

24 André Breton (1979: 66).

25 Siehe hierzu die berühmten Briefe Rimbauds an Georges Izambard (13. Mai 1871) bzw. Paul Demeny (15. Mai 1871); Rimbaud (1979: 248-254).

26 Ob man darum - aus dem Grund größerer Nähe zur äußeren Wirklichkeit in Un barbare en Asie eine unterschiedliche Wertung beider Reisebücher vornehmen kann, sei allerdings bezweifelt. Bruno Thibault nimmt eine solche Wertung vor: »Ecuador n'est pas un chef-d'œuvre; c'est un cahier d'esquisses, une recherche inquiète du vvraic voyage. Toutefois ce journal de voyage marque une étape importante, celle de la négativité, que l'auteur saura dépasser dans son voyage suivant«, in: Bruno Thibault (1990: 487). Einem antiklassischen Autor par excellence wie Michaux das Skizzenhafte eines Buchs zum Vorwurf zu machen, erscheint uns ebenso absurd wie der Versuch, die Negativität, 
der Autor dabei Impulse des Denkens seiner Zeit insofern auf, als er den Blick auf die Dinge als Blick auf deren Entstehung im Subjekt versteht und die Wahrnehmung als ein prozessuales, immer wieder in Fluss gebrachtes »Wahrnehmungserlebnis« (Edmund Husserl) auffasst (auf Michaux' Nähe zu Merleau-Ponty als dem Vertreter der französischen Phänomenologie und dessen Versuch, einen präobjektiven Zugang zur Welt zu denken, haben wir - ebenso wie auf seine Affinität zu der von verschiedenen Theoretikern betriebenen Kritik des autonomen Ich - bereits hingewiesen). Indem das Ich in einem Kontinuum sich ändernder Aufmerksamkeitsrichtungen steht und seinen geistigen Blickstrahl über die Gegenstände wandern lässt, entsteht - weil der Horizont der Wahrnehmungen stets offen bleibt - neben der aktuellen Wahrnehmung zugleich auch ein Feld von Gegenständen, die prinzipiell ins Bewusstsein treten können, aber zur Zeit vom Bewusstsein nicht fokussiert werden: $»$ Das Bewusstsein der Aktualität wird [...] von einem Hof von Inaktualitäten umgeben. $\ll^{27}$ Der Autor - der den Philosophen natürlich den Humor voraus hat - beschreibt, wie sich das Schiff der Insel Curaçao nähert. Man fährt mehrere Stunden lang in einiger Entfernung zur Insel, um dann direkt in den Hafen einzulaufen. Hierbei nun bemerkt der Autor eine Störung seiner Wahrnehmung: Seine Sinne sind noch immer auf die Eintönigkeit des Meeres eingestellt und mit einer ganzen, von Leben wimmelnden Stadt überfordert. Das Bewusstsein zeigt seine Grenzen: Es kann die Ganzheit nicht wahrnehmen, sondern müsste Stück für Stück, kontinuierlich und additiv verfahren, um sich allmählich ein Bild zu machen. Am ersten Tag würde ein einziges Haus mit Tür und Vordach ausreichen, am zweiten könnte ein Kind mit einem Sandeimer dazutreten; dies wäre der dem Bewusstsein angemessene Umgang mit Vielfalt. Nach meinem Dafürhalten haben wir es hier mit einer der schönsten Liebeserklärungen zu tun, die ein reisender Phänomenologe an die Welt der Dinge abzugeben imstande ist ${ }^{28}$ :

die hier den Motor eines gesamten - literarischen wie bildnerischen - Lebenswerks bildet, als vorübergehende, noch nicht wesentliche Phase darstellen zu wollen.

27 Siehe Werner Marx ( $\left.{ }^{2} 1987: 59\right)$.

28 Obwohl bzw. gerade weil sich das phänomenologische Ich als »uninteressierter « und »unbeteiligter Zuschauer « versteht (siehe Edmund Husserl, Cartesianische Meditationen, § 15), hat der Phänomenologie in der Debatte zumindest eine ethische Komponente zugesprochen werden können. Die Reduktion auf das Bewusstseinsleben bedeutet eine "Schwächung der Subjektivität ( Pier A. Rovatti [1989: 281]), das heißt eine "Bewegung des Innehaltens gegenüber dem Nichtbeherrschbaren« (ibid.: 280) und führt zur "Gegenwart eines vorprädikativen Erlebnisses« (ibid.). "Die Retrozendenz als ethische Bewegung [...] ist ineins eine Entscheidung und ein Geschehenlassen; die Verantwortung, die das phänomenologische Tun mit sich bringt, zielt in eine Richtung, die der Selbstbehaup- 


\section{Eberhard Geisler}

Curaçao à tribord.

On longe l'île, de loin, des heures durant.

Puis tout à coup on a mis le cap sur l'entrée du port. On est entouré, à moins d'une encâblure, de toutes choses, et notre œil ne voit rien, et notre cerveau ne comprend rien.

Ils restent marins encore quelque temps. La cristallisation se fait trop rapide. On nous eût encore donné une seule maison dans l'Atlantique, une porte et un auvent, et, le jour suivant un bébé près d'un seau de sable, quelle joie!

Mais non, on me prive de tout pendant quinze jours, puis en une minute toute une ville se pousse contre moi, des maisons par centaines, des hangars, des cheminées [...] des constructions inconnues, je ne sais quoi encore, et je ne sais qu'en faire. (EJV, 25-26)

\section{Bibliographie}

Literarische Werke und andere Quellen

Breton, André (1979 [1924]): Les pas perdus, Paris: Gallimard.

Da Vinci, Leonardo (1928): Prose, hrsg. v. Luigi Negri, Turin: Unione Tipogràfico-Editrice Torinese.

Gide, André ( ${ }^{60} 1947$ [1927]): Voyage au Congo. Carnets de route, Paris: Gallimard.

Michaux, Henri (1980 [1954]): Face au verrous, nouvelle édition revue et corrigée, Paris: Gallimard.

Michaux, Henri (1982 [1933]): Un barbare en Asie, nouvelle édition revue et corrigée, Paris: Gallimard.

Michaux, Henri ( ${ }^{2} 1982$ [1951]): Mouvements, Paris: Gallimard.

Michaux; Henri (1983 [1938]): Plume, précédé de Lointain intérieur, Paris: Gallimard.

Michaux, Henri ( $\left.{ }^{2} 1983\right)$ : »Quelques renseignements sur cinquante-neuf années d'existence «, Cahiers de l'Herne: Henri Michaux (1966), Paris: L'Herne, pp. 11-15.

Michaux, Henri (1984 [1929]): Ecuador. Journal de voyage, nouvelle édition revue et corrigée, Paris: Gallimard.

tung eines herrschaftsausübenden Subjekts entgegengesetzt ist«, (ibid.: 283). »Das Phänomen, das zu ssehen ‘ die Phänomenologie durch die epoché erreichen will, erweist sich als sunsagbar« « (ibid: 286). Die Prozessualität des phänomenologischen Erkennens schließlich trägt gleichfalls dazu bei, den Anspruch, das Ganze je abschließend zu sehen, aufzugeben, und würdigt den Gegenstand einer permanenten Annäherung (cf. ibid: 288). Differenzierend sei noch darauf hingewiesen, dass sich das Bewusstsein für Husserl durchaus auch auf einen kollektiven Gegenstand richten kann. Als Beispiel für dieses vielstrahlig Bewusste nennt er - im Zusammenhang seiner phänomenologischen Beschreibungen der verschiedenen Intentionalitäten des Bewusstseins - die Mutterliebe: »[...] die Mutter, die liebend auf ihre Kinderschar blickt, umfaßt in e i n e m Akte der Liebe jedes Kind einzeln und alle zusammen«, in: Edmund Husserl $\left({ }^{4} 1980: 251\right)$. Das von Husserl gewählte Beispiel macht jedoch deutlich, dass der Zerteilung des vom Ich ausgehenden Liebesstrahls in ein »Bündel von Strahlen« bzw. dem Akt der Wahrnehmung eines kollektiven Objekts ein jeweiliges Kennen- und Liebenlernen der Einzelobjekte (hier: der kindlichen Individuen) vorausgegangen sein muss. 
Michaux, Henri (1985): »Musique en déroute«, in: id.: Déplacements. Dégagements, Paris: Gallimard, pp. 25-35.

Morand, Paul (1926): Rien que la terre, Paris: Grasset.

Rimbaud, Arthur (1979): CEuvres complètes, hrsg. v. Antoine Adam. Paris: Gallimard.

\section{Forschungsliteratur}

Bellour, Raymond (1986): Henri Michaux, Paris: Gallimard.

Geisler, Eberhard (1992): Henri Michaux. Studien zum literarischen Werk, Stuttgart / Weimar: Metzler.

Heißenbüttel, Helmut (1966): „Anmerkungen zu einer Literatur der Selbstentblößer (M. Leiris, A. Breton, H. Michaux)«, Merkur 20, pp. 568-577.

Husserl, Edmund ( $\left.{ }^{4} 1980\right)$ : Ideen zu einer reinen Phänomenologie und phänomenologischen Philosophie, Tübingen: Niemeyer.

Martin, Jean-Pierre (1991): »De la lecture comme sabotage. Michaux et Papazoff «, Poétique 88, pp. 399-418.

Marx, Werner ( 1987$)$ : Die Phänomenologie Edmund Husserls. Eine Einführung, München: Fink.

Merleau-Ponty, Maurice (1969): »L'expression et le dessin enfantin«, in: id.: La Prose du monde, Paris: Gallimard, pp. 204-211.

Meschonnic, Henri (1987): »Le rythme et le poème chez Henri Michaux«, in: Mathieu, Jean-Claude / Collot, Michel (Hrsg.): Passages et langages de Henri Michaux. Actes de la troisième »Rencontre sur la poésie moderne« (ENS, juin 1986), Paris: Corti, pp. 185-208.

Moura, Jean-Marc (1998): La Littérature des lointains. Histoire de l'exotisme européen au XXe siècle, Paris: Champion.

Rabaté, Étienne (1987): »L'enfance«, in: Mathieu, Jean-Claude / Collot, Michel (Hrsg.): Passages et langages de Henri Michaux. Actes de la troisième »Rencontre sur la poésie moderne « (ENS, juin 1986), Paris: Corti, pp. 117-130.

Rovatti, Pier Aldo (1989): »Das Rätsel der Epoché«, in: Jamme, Christoph / Pöggeler, Otto (Hrsg.): Phänomenologie im Widerstreit. Zum 50. Todestag Edmund Husserls, Frankfurt am Main: Suhrkamp, pp. 277-288.

Thibault, Bruno (1990): ") Voyager contre de voyage d'Henri Michaux«, The French Review 63: 3, pp. 485-491. 